\title{
REMARKS ON DECOMPOSITIONS OF CATEGORIES
}

\author{
JOHN R. ISBELL
}

Consider, to begin with, decompositions of complete categories as products or as coproducts. However, a complete category cannot be a coproduct of two or more nonempty categories. In fact, there is a well-known finest decomposition of any category as a coproduct, whose factors are called the (weak) components and are not connected by any morphisms. (Thus, for instance, objects in different components have no product object.) However, one can define strong components (as in graph theory) so that for two objects $X, Y$ in the same strong component, both $\operatorname{Hom}(X, Y)$ and $\operatorname{Hom}(Y, X)$ are nonempty.

Two theorems will be proved. A strong component of a complete category has a unique completion; two direct decompositions of a skeletal complete category have a common refinement.

For fine points of terminology we refer to [1]; but a reader will lose little if he simply considers that all categories to be decomposed belong to a given Grothendieck universe whose cardinal number is called $\infty$, small means "smaller than $\infty$," and small-complete means "having limits and colimits of arbitrary small diagrams." (The other completeness notions mentioned are slightly stronger but equivalent for well-powered co-well-powered categories.) Of course results from [1] are used.

Precisely, we call a category e strongly connected if no (hom)" set $\mathfrak{e}(X, Y)$ is empty. The maximal strongly connected subcategories are called strong components. The strong components partition the objects, of course. They form a skeletal schlicht (partially ordered) category $\mathrm{Sc}(\mathfrak{e})$, where by definition there is a unique morphism from $Q$ to $B$ in $\operatorname{Sc}(\mathcal{C})$ if $\mathfrak{e}(A, B)$ is nonempty for (arbitrary) representative objects $A \in|a|, B \in|B|$; otherwise there is no morphism from $a$ to $B . \mathrm{Sc}(\mathbb{C})$ is a reflection of $\mathcal{C}$ in the subcategory of Cat composed of skeletal schlicht categories.

Obviously (from [1]), for schlicht categories, completeness is equivalent to small-completeness. Obviously:

Proposition 1. If e is small-complete so is $\mathrm{Sc}(\mathfrak{e})$. A small-complete category is strongly connected if and only if it has a two-sided zero.

(The morphisms between left and right zeros are isomorphisms be-

Received by the editors April 10, 1967. 
cause the compositions can only be identities; one-sided zeros have no endomorphisms.)

We call a category e conditionally left small-complete if for every

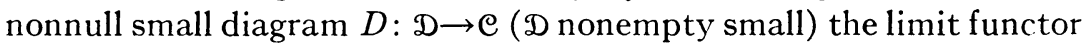
of $D$ either is representable or has all its values empty. If $\mathfrak{C}$ is strongly connected, it is equivalent to require that every small nonempty family of objects has a product and every coterminal pair of morphisms $X \rightarrow Y$ that is equalized by some morphism into $X$ has an equalizer. Obvious modifications give the definitions (with some equivalent variants) of conditionally left (strict-, wide-) complete, the dual notions, and the two-sided notions. Any coproduct of conditionally complete categories is conditionally complete.

Completing a conditionally complete category is a trivial matter (Theorem A). In fact, this is true for one-sided completion, but there are several cases and we omit checking. The basic constructions are two (and their duals), as follows: Adjunction of a detached left zero to $\mathfrak{C}$ is full embedding of $\mathfrak{e}$ in a category which has one more object $L$, with every $\operatorname{Hom}(L, X)$ having one element and every $\operatorname{Hom}(X, L)$, for $X \neq L$, no element; conditional adjunction of a detached left zero is the same, if $\mathfrak{C}$ lacks a left zero, but $1: \mathfrak{C} \rightarrow \mathfrak{C}$ if $\mathfrak{C}$ has a left zero.

TheOREM A. The following properties for a category $\mathbb{C}$ are equivalent.

(i) $\mathcal{e}$ is conditionally small-complete.

(ii) Adjunction of detached left and right zeros to $\mathfrak{e}$ yields a smallcomplete category.

(iii) e has a unique small-completion (up to equivalence) obtained by conditional adjunction of detached left and right zeros.

Proof. Assuming (i), and adjoining detached left and right zeros $L$ and $R, R$ is the product of the empty family and of any family of copies of $R$. The product of any family that includes $L$ is $L$. For a family $F$ including objects of $\mathfrak{e}$ and including no copy of $L$, let $F^{\prime}$ be the subfamily in $\mathcal{e}$ (i.e. ignore $R$ ). If $F^{\prime}$ has a product $P$ in $\mathcal{e}, P$ remains the product of $F$; otherwise $L$ is the product. Two distinct coterminal maps must be in $\mathfrak{C}$; if they have an equalizer in $\mathfrak{e}$, it remains their equalizer, and otherwise $L$ is their equalizer.

Assuming (ii), note that if $\mathfrak{C}$ has a left zero $L_{1}$, the new left zero is not a limit of any diagram in $\mathcal{C}$. Nor is it a colimit except of the empty diagram, which already had the colimit $L_{1}$ in $\mathfrak{C}$. Then plainly the construction of (iii) yields one completion $\mathcal{E}$. To prove uniqueness it suffices to show that if $\mathcal{C}$ lacks a left zero then the left zero in any completion $D$ is detached, and dually. Assume, on the contrary, that there is a map $p: X \rightarrow M$ from some $X$ in $\mathcal{C}$ to the left zero of $D$. There 
is also $i: M \rightarrow X$, and $p i: M \rightarrow M$ must be $1_{M}$. Thus $r=i p: X \rightarrow X$ is idempotent. Evidently $r$ cannot split in $\mathfrak{C}$, as the splitting in $\mathfrak{D}$ is unique and $\mathcal{C}$ lacks a left zero. Then $r$ cannot split in $\mathcal{E}$ either, for the added objects are detached. The contradiction, and duality, establish (iii).

The implications (iii) $\Rightarrow$ (ii) $\Rightarrow$ (i) are trivial.

REMARKs. (a) If throughout Theorem A "small-" is replaced by "strict-," "extremal-," or "wide-," the result holds, by a trivially modified proof.

(b) Clause (ii) above describes a reversible procedure; removal of whichever zeros of a complete category are detached leaves a conditionally complete category.

For the (generalized) application to strong components, see Proposition 3.

Commonly the unique completion of a conditionally complete category is a normal completion [1]. There are trivial counterexamples, and substantially no others. Let w denote the partially ordered category of small ordinal numbers. Let us say that $W$ is finally embedded in $\mathfrak{C}$ if it is fully embedded so that each object of $\mathfrak{C}$ has at most one map to any object of $W$, and at least one map to some object of W.

Proposition 2. A conditionally complete ordinary category $\mathfrak{e}$ has a nonnormal completion if and only if $\mathfrak{W}$ is finally embedded in $\mathfrak{e}$ or in $\mathfrak{e}^{*}$.

Proof. If $W$ is finally embedded in $\mathcal{C}$, then every small family of objects has a nonempty-valued coproduct functor; thus, if it is nonempty, it has a coproduct in $\mathfrak{C}$. Each object $X$ of $\mathfrak{e}$ maps to some $W$ in $W$, and then all strict or (by induction) multistrict quotients of $X$ map to $W$. Since $W$ is fully and finally embedded, the right zero $R$ of the completion is not in $\mathfrak{C}$; so it is not in the right closure of $\mathfrak{C}$. Thus on this hypothesis or the dual one, the completion is not normal. (e need not be ordinary for this half.)

If $\mathcal{C}$ is ordinary and Theorem $\mathrm{A}$ applies, and the completion $\mathcal{E}$ is not normal, then we may assume the right zero $R$ is not in the right closure of $\mathfrak{e}$ (else the dual holds). Each object $X_{\alpha}$ of $\mathfrak{e}$ has a smallest multistrict quotient $V_{\alpha}$ in $\varepsilon$, and it is not $R$. If there were two distinct coinitial maps into $V_{\alpha}$ there would be a smaller multistrict quotient. Let $W_{\alpha}$ be the smallest multistrict quotient of the coproduct of all $X_{\beta}(\beta<\alpha)$. There may be repetition $\left(W_{\alpha+1}\right.$ isomorphic with $\left.W_{\alpha}\right)$, but the skeleton of these $W$ 's has to be 'W.

The next proposition says that strong components of a more or less conditionally complete category are similarly complete and the embeddings are similarly conservative. 
Proposition 3. For every nonnull diagram $D$ in a strong component a of a category $\mathcal{C}$, if $D$ has a limit object $Y$ in $\mathfrak{C}$ then $Y$ is in $a$ or the limit functor of $D$ in a is empty-valued.

Proof. If the limit functor is not empty-valued then some $X$ in $Q$ maps to $Y$; and $Y$ maps to some $D(Z)$ in $Q$.

This has the suggested application to extremal- and wide-, as well as small- and strict-completeness, because of the limit characterizations of monomorphisms and extremal monomorphisms. $(X \rightarrow Y$ is monomorphic if and only if the pullback of $X \rightarrow Y \leftarrow X$ is $X$. Extremal monomorphisms are multistrict [1].)

How do the strong components of a complete category fit together? If they are complete, i.e. they have two-sided zeros, then subject to boundedness conditions they are connected by pairs of adjoint functors; when $Q \rightarrow B, B$ should be reflective and $a$ coreflective in the full category on $Q \cup B$, and if they are, the reflector and the coreflector will yield adjoint functors between $Q$ and $B$. Working this out is an easy exercise, and rather useless. Familiar examples of complete categories that are not strongly connected do not have complete strong components. The simplest example is the (small) algebras with one unary operation. It is worth a moment to look at why only one of the $\infty$ strong components is complete, since the argument applies to almost any category of sets with structure (e.g., as in [2]). $X$ and $X \times X$ are always in the same strong component; if $X$ is a relative zero (two-sided, or right), the two coordinate projections of $X \times X$ must be identical. Then $X$ has at most one element. For the algebras mentioned, the one with exactly one element is a right zero of its component $R$, but $R$ has no left zero. As it happens, no algebra not in $R$ has a reflection in $R$. I know nothing of the strong component structure of this example.

It is natural to ask how completion of a category $\mathfrak{C}$ is related to completion of its strong components and of $\mathrm{Sc}(\mathcal{e})$; but there seems to be no substantial relation beyond Propositions 1 and 3 . It is true that every completion $\mathcal{E}$ of a genuine coproduct $\mathcal{C}$ of categories $\mathfrak{C}_{\alpha}$ (at least two nonempty $\mathfrak{C}_{\alpha}$ occurring) has detached zeros, and with those removed it reduces to a coproduct of conditional completions $\varepsilon_{\alpha}$ of the $\mathfrak{C}_{\alpha}$. To prove this, define $\mathcal{E}_{\alpha}$ as the full subcategory on the objects $Y$ such that maps $X \rightarrow Y \rightarrow Z$ exist with $X, Z$ in $\mathcal{e}_{\alpha}$. Evidently the $\varepsilon_{\alpha}$ are disjoint and there is no map between them. An object $T$ of $\varepsilon$ that is in no $\mathcal{E}_{\alpha}$ has either $\mathfrak{e}(T, \quad)$ or $\mathfrak{e}(, T)$ empty-valued; and the zeros are such objects. Thus the coproduct of the $\varepsilon_{\alpha}$ is conditionally complete, and the zeros complete it. If some $\varepsilon_{\alpha}$ had a conditionally complete full proper subcategory $\mathbb{D}$ containing $\mathfrak{C}_{\alpha}$, replacing $\varepsilon_{\alpha}$ with 
$\mathbb{D}$ in the construction of $\varepsilon$ would still give a complete extension of $\mathbb{C}$.

A third unique decomposition, for complete categories and up to equivalence, is direct decomposition as a product in Cat. There is uniqueness for finite decompositions; infinite direct decompositions (already for complete lattices) may not go all the way to atoms, but at least any two have a common refinement.

Theorem B. Any two direct decompositions of a skeletal category with right zero have a common refinement.

Proof. Given an identification $\varepsilon=\times Q_{\alpha}$, the coordinates $0_{\alpha}$ of the right zero 0 are right zeros of the factors since each $\mathcal{E}(X, 0)$ is a Cartesian product set of power 1 . Each $Q_{\alpha}$ can be embedded in $\mathcal{E}$ so that the $\alpha$ th coordinate projection is the identity and the others take all of $a_{\alpha}$ to $0_{\beta}$. Then $Q_{\alpha}$ becomes a full subcategory of $\mathcal{E}$ such that every direct factor of an object of $a_{\alpha}$ is in $Q_{\alpha}$ and every product of objects of $a_{\alpha}$ that happens to exist in $\mathcal{E}$ is in $a_{\alpha}$. Moreover, many products exist: every product of a family of objects chosen one from each $Q_{\alpha}$. These exhaust $\mathcal{E}$, the maps between two such products are just those specified by maps between corresponding factors, and a map is determined by its coordinates. The description just given for a family of subcategories $Q_{\alpha}$, conversely, holds only when $\mathcal{E}$ is isomorphic with a product and the factors are embedded as indicated. Then for another such decomposition of $\mathcal{E}$ as $\times \mathfrak{e}_{\gamma}$, since the $\mathfrak{e}_{\gamma}$ are closed under direct division and conditionally closed under direct product, each $\mathfrak{C}_{\gamma}$ is $\times\left(Q_{\alpha} \cap \mathfrak{e}_{\gamma}\right)$.

By a (full) subdirect decomposition of $\mathcal{E}$ we shall mean a (full) embedding of $\mathcal{E}$ in a product $\times a_{\alpha}$, one-to-one on objects. Nonfull decompositions may be of interest, but they seem to have none of the uniqueness that we are looking for just now. Uniqueness for full subdirect decompositions is strongly suggested by the observation that, at least in the finite case, they must actually be direct whenever the following argument applies. If $\varepsilon \subset Q \times B$ includes $\left(A, B_{1}\right)$ and $\left(A_{1}, B\right)$, then it should include the product $\left(A \times A_{1}, B \times B_{1}\right)$, and $(A, B)$ should arise as a retract. (Of course, this makes $\varepsilon$ the product, not necessarily of $Q$ and $B$, but of its coordinate projections. In the general situation $\varepsilon \subset \times a_{\alpha}$, the factors of the subdirect decomposition are the smallest subcategories of the $Q_{\alpha}$ that contain the projections of $\mathcal{E}$. If $\varepsilon$ is fully embedded, still its projections may not be subcategories, because of empty hom sets.)

Proposition 4. Finite full subdirect decompositions of a strongly connected skeletal category with products of pairs of objects and in which idempotents split are direct decompositions and have common refinements. 
Proof. The hypotheses suffice for the proof (indicated above) that the decompositions are direct. To reduce notation we consider a binary decomposition $\varepsilon=Q \times Q$ and another full subdirect decomposition $\varepsilon \subset \times \mathfrak{e}_{i}$, which need not be finite. Clearly it will suffice to show that the congruence relation induced by a projection $\pi$ of $\mathcal{E}$ upon one of its factors $\mathcal{C}_{i}$ is the product of congruence relations on $a$ and $B$.

The rest of the proof uses the facts that $\pi$ is a full functor preserving products and that $\mathfrak{C}_{i}$ is skeletal, besides products and $\mathcal{E}=a \times B$. Three things must be proved. (1) If $\pi(\alpha, \beta)=\pi\left(\alpha^{\prime}, \beta^{\prime}\right)$ for coterminal $(\alpha, \beta),\left(\alpha^{\prime}, \beta^{\prime}\right)$, then $\pi\left(\alpha^{\prime}, \beta\right)$ is the same. (2) Once $\pi(\alpha, \beta)=\pi\left(\alpha^{\prime}, \beta\right)$, $\pi(\alpha, \gamma)=\pi\left(\alpha^{\prime}, \gamma\right)$ for all $\gamma$. (3) If $\pi(\alpha, \beta)$ is an identity, so is $\pi\left(\alpha, 1_{B}\right)$ for all $B$. There are five pieces of computation. The first turns on the fact that an exchange of coordinates $\sigma$ in $A \times A$ gives an automorphism $\hat{\sigma}=(\sigma, 1)$ of $(A \times A, B \times B)=(A, B) \times(A, B)$ leaving the diagonal fixed. Hence $\pi(\hat{\sigma})$ does not change any map into $\pi(A, B)$ $\times \pi(A, B)$ that factors through the diagonal, i.e., the switch $\hat{\sigma}$ in quadruples $\left(\alpha, \beta, \alpha^{\prime}, \beta^{\prime}\right)$ diagonalized by $\pi$ is wiped out by $\pi$; this is (1). For (2), the basic idea is that maps of triple products taking a "point" $(x, y, z)$ to $(\alpha(x), \beta(y), \gamma(z))$ or $\left(\alpha^{\prime}(x), \beta(y), \gamma(z)\right)$ coincide; the idea works if a dummy fourth variable from $A$ is introduced. For (3), first, if $(A, B)$ and $\left(A^{\prime}, B^{\prime}\right)$ have the same image $C$, and one writes out the morphisms, one sees at once that the images $C^{\prime}, C^{\prime \prime}$ of $\left(A, B^{\prime}\right)$ and $\left(A^{\prime}, B\right)$ have $C$ as a retract. But also $C^{\prime} \times C^{\prime \prime}=C \times C$, whence isomorphisms. Since $\mathfrak{e}_{i}$ is skeletal, $C=C^{\prime}=C^{\prime \prime}$. Next, one proves, as in (2), that if $\pi\left(\alpha, 1_{B}\right)$ is an identity for some $B$ it is so for all $B$. This gives the ambiguous symbol $(\alpha, 1)$ enough meaning for the following. One has an automorphism $\theta$ of $C$ that is the image of a map $(\alpha, 1)$, with $\theta^{-1}$ the image of a map $(1, \beta)$. Since $\alpha$ 's and $\beta$ 's commute and generate everything, $\theta$ commutes with all automorphisms of $C$. So does the automorphism of $C \times C$ with $\operatorname{coordinates} \theta$ and 1 ; exchanging coordinates, $\theta=1$.

On replacing "finite" by "small," I suspect that it can be done without assuming infinite products (though of course the decompositions would not be direct); but the proof given requires the products, and all the shuffling for (1), (2), and (3) must be adapted to changing sets of coordinates.

\section{REFERENCES}

1. J. R. Isbell, Structure of categories, Bull. Amer. Math. Soc. 72 (1966), 619-655.

2. O. Wyler, Operational categories, Proceedings of the Conference on Categorical Algebra (La Jolla, 1965), Springer, New York, 1966. 